\title{
Caesarean Section During Second Stage of Labor in a Tertiary Centre
}

\author{
Padma Gurung, ${ }^{1}$ Sameer Malla, ${ }^{1}$ Sushma Lama, ${ }^{1}$ Anagha Malla, ${ }^{1}$ Alka Singh ${ }^{1}$ \\ 'Department of Obstetrics and Gynecology, Patan Hospital, GPO 24900, Lalitpur,Nepal.
}

\begin{abstract}
Background: There is an alarming rise in caesarean section leading to increased adverse outcomes for both the mother and fetus when compared with vaginal delivery. Within this increasing caesarean section rate, there is a concerning increase in the rate of second stage caesarean section. This study highlight the feto-maternal outcome of caesarean section in second stage of labour.

Methods: This was a retrospective cohort review of all women with a singleton, cephalic fetus at term delivered by caesarean section in the second stage of labor between April 1, 2013 and March 30, 2017 at Patan Academy of Health Sciences. The main outcome measures were second stage caesarean section, indications and its maternal and fetal morbidity.

Results: During the study period, there were 40,860 deliveries. A total of 18,011 (44\%) babies were born by caesarean section, 10484 emergency and 7527 elective. Out of the emergency caesarean section, $200(1.9 \%)$ were performed in second stage of labor. In this study, the most common indication was cephalopelvic disproportion. $(92.4 \%)$ were delivered without a trial of instrumental delivery. In terms of maternal complications, atonic post partum haemorrhage uterine incision extension $18(12.5 \%)$, postoperative fever $27(18.8 \%)$, wound infection $7(4.8 \%)$ were observed. In perinatal complications, meconium stained amniotic fluid 49(34.2\%), neonatal hyperbilirubinemia $14(9.7 \%)$ and increased nursery admission $2(15.3 \%)$ and $2(1.3 \%)$ perinatal mortality were seen.

Conclusions: Cesarean sections done in second stage of labor are associated with several intra-operative maternal complications and neonatal morbidity.

Keywords: Caesarean complications; emergency caesarean section; maternal morbidity; neonatal morbidity; second stage of labor.
\end{abstract}

\section{INTRODUCTION}

Caesarean section is the most commonly performed major abdominal surgery in women. Despite efforts to limit operative abdominal deliveries, there is an alarming rise in Caesarean section (CS) leading to increased adverse outcomes for both the mother and fetus when compared with vaginal delivery. ${ }^{1}$ Within this increasing $\mathrm{CS}$ rate, there is a concerning increase in the rate of second stage cesarean section. ${ }^{2}$ Recent decline in the use of instrumental delivery, ${ }^{1}$ a combination of lack of training and supervision for junior staff in secondstage decision-making, a loss of technique associated with difficult-assisted delivery ${ }^{2}$ and concerns relating to maternal and neonatal morbidity with associated litigious issues might have contributed to this disturbing trend.
Second stage caesarean section is associated with increased maternal as well as fetal complications as it is technically difficult to perform because of the deeply impacted fetal head in the pelvis and the presence of thinned out edematous lower segment. ${ }^{3}$

The objective of this study is to review the second stage cesarean deliveries at Patan Hospital, their indications and associated maternal and perinatal complications.

\section{METHODS}

This retrospective study assessed all caesarean sections performed at full cervical dilatation between 1 April 2013 and 31 March 2017 at Patan Hospital, Patan Academy of Health Sciences. Caesarean section cases were identified through the operating theatre data log. 
The medical record, specifically the record of labor and operation reports, was reviewed for all CS cases over the study period. Women with a singleton fetus in cephalic presentation at term ( $\geq 37$ weeks) who underwent CS at full dilatation were included. Multigravida with comorbid conditions like diabetes and preeclampsia were excluded. These second stage Caesarean sections were analysed in terms of indications, instrumentation before caesarean section, intra operative complications like haematuria, uterine incision extension, atonic post-partum haemorrhage (PPH), postoperative complications like febrile illness, wound infection and neonatal morbidity and mortality. All the data collected were pooled together and recorded and entered in master chart. Data analysis was done using SPSS version 17. Permission for the study and ethical approval wasobtained from the institutional review committee (IRC) of Patan Academy of Health Sciences (PAHS).

\section{RESULTS}

During the index period, a total of 18011 women delivered by caesarean section, 10484 emergency and 7527 elective cases. Of these 200 (1.9\%) were at full cervical dilatation, $>37$ weeks gestation with a singleton fetus in cephalic presentation. Fifty cases were excluded and seven medical records could not be retrieved.

Figure 1 illustrates the trend of CS deliveries and normal vaginal deliveries over the time period examined. During the study period the caesarean section rate remained at a constant high, $44.5 \%$ in 2013 to $45 \%$ in 2016.

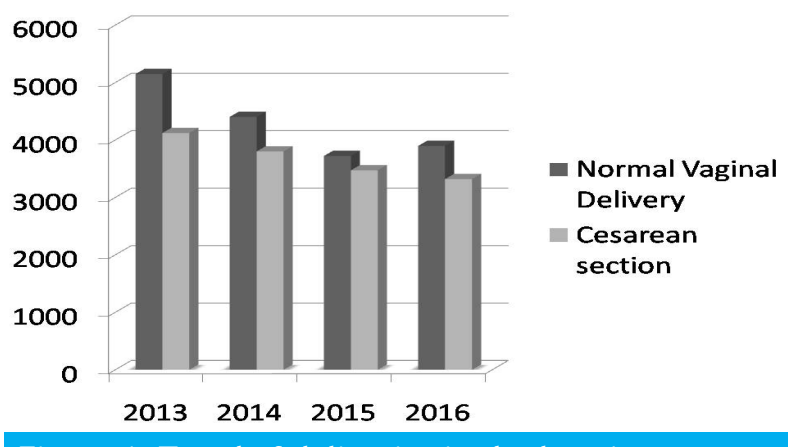

Figure 1. Trend of deliveries in the last 4 years.

Figure 2 illustrates the trend of second stage Caesarean section and instrumental delivery in the last 4 years. The rate of second stage Caesarean decreased from $4.2 \%$ $(\mathrm{n}=92)$ in 2013 to $0.95 \%(\mathrm{n}=21)$ in 2017 . During the study period the rate of instrumental deliveries increased from $0.1 \%(n=49)$ in 2013 to $0.3 \%(n=114)$ in 2017 with an overall instrumental delivery rate of $1.1 \%$.

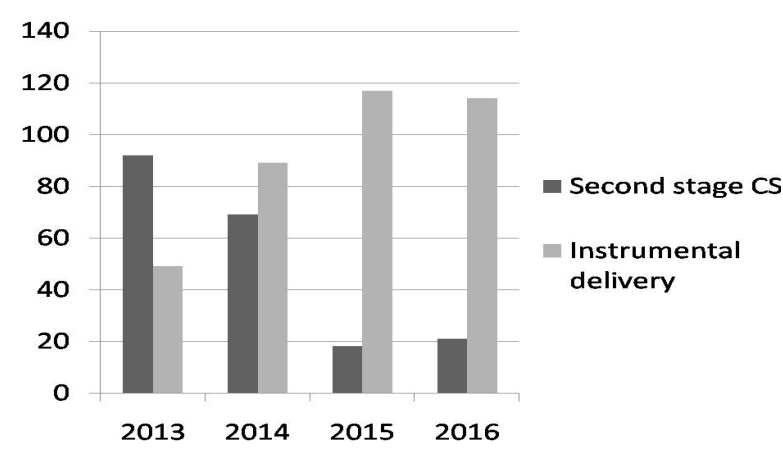

Figure 2. Trend of second stage Caesarean section and instrumental delivery in the last 4 years.

Cephalo-pelvic disproportion (CPD) and fetal distress were the most common indications for CS in the second stage $(53.8 \%$ and $34.9 \%$ respectively) followed by failed instrumental delivery (7.6\%). Five (3.4\%) declined trial of operative vaginal delivery and opted for second-stage CS. Mean duration of surgery was 57.68 min and mean hospital stay was 5.59 days.

Table 1 summarises the maternal operative complications and table 2 outlines the perinatal complications.

Operative complications associated with emergency CS in second stage of labor, at PAHS 2013-2017.

\begin{tabular}{|lrr|}
\hline Table 1. Maternal complications. & & \\
\hline Maternal Complications & Number & Percentage \\
\hline Atonic PPH & 7 & $4.8 \%$ \\
\hline Uterine incision extension & 18 & $12.5 \%$ \\
\hline Postoperative fever & 27 & $18.8 \%$ \\
\hline $\begin{array}{l}\text { Wound infection requiring } \\
\text { resuturing }\end{array}$ & 7 & $4.8 \%$ \\
\hline
\end{tabular}

\section{Table 2. Fetal and newborn complications.}

\begin{tabular}{|lrr|}
\hline Perinatal complications & Number & Percentage \\
\hline Meconium stained liquor & 49 & $34.2 \%$ \\
\hline Admission to nursery & 22 & $15.3 \%$ \\
\hline NICU admission & 5 & $3.4 \%$ \\
\hline Neonatal jaundice & 14 & $9.7 \%$ \\
\hline Cephalhematoma & 2 & $1.3 \%$ \\
\hline Apgar score $<7$ at 5 min & 13 & $9.0 \%$ \\
\hline Fresh still birth & 1 & $0.6 \%$
\end{tabular}

Mean weight of the babies of the second stage caesarean section was $3.4 \mathrm{~kg}$. Five (3.4\%) babies were admitted to the Neonatal Intensive Care Unit and 22 (15.3\%) to neonatal nursery for management of respiratory distress, sepsis, jaundice, and observation. Four (2.7\%) 
babies were delivered by breech extraction at CS due to difficulty in delivering the deeply impacted fetal head.

Of the study population, 104 (72.7\%) second stage CS was operated by gynaecologists and 39 (27.2\%) by medical officers. There were no significant differences in the operating time, incidence of extensions, febrile morbidity, duration of hospital stay and adverse fetal outcome in the surgeries done by medical officers when compared to the obstetricians.

\section{DISCUSSION}

The international literature ${ }^{2,4}$ suggests that within a rising CS rate, there is an increasing trend to perform CS at full cervical dilatation. The strong medico-legalmind set in current obstetrics, and concerns over neonatal and maternal morbidity associated with difficult or failed instrumental delivery may contribute to this trend. ${ }^{2}$

Over the 4-year study period, the overall CS rate was higher than international rates. ${ }^{2,4}$ This higher rate of CS might be because Patan hospital is a referral centre where high-risk patients from surrounding districts are referred, mostly for operative deliveries. However, our rates of CS at full cervical dilatation are lower than other published cohorts. ${ }^{4}$ The lower rate may be explained by more women not reaching full dilatation due to an arrest in the first stage of labor or unsuccessful induction of labor.

Caesarean section in the second stage of labor is a technically difficult operation with distortion of pelvic anatomy and thefetal head that is often deeply impacted in the maternal pelvis. Women delivered by CS at full dilation have a higher riskof obstetric haemorrhage, bladder injury, extended uterine tear leading to broad ligament hematoma, infection and longer hospital stay. ${ }^{3}$ A retrospective study from Canada has shown that women delivered by Caesarean sections at full dilatation of the cervix were 2.6 times likely to have intraoperative traumatic complications. ${ }^{3}$ In our study uterine incision extension was seen in $12.58 \%$, which is slightly higher compared to the other studies. ${ }^{5,6}$ This might be due to the fact that the most common indication of second stage in our study was cephalopelvic disproportion with major caput and moulding formation making the delivery of the fetal head challenging. The most common maternal operative complications seen in our study was blood stained urine in $27(18.8 \%)$, febrile illness in 27 (18.8\%), and wound infection in seven $(13.9 \%)$ cases.

Atonic postpartum haemorrhage was seen in $7(4.8 \%)$ cases, which is slightly less than in the previous studies..$^{5,6}$ The use of prophylactic uterotonics in second stage Caesarean could have contributed to this decreased number. One woman returned to the operating room for management of postpartum haemorrhage. The rest of the PPH cases were managed with uterotonic drugsanduterovaginal packing. Four $(2.7 \%)$ of these women required blood transfusion.

Controversies regarding the fetal outcome in the cases of caesarean sections in second stage of labor are seen throughout literature. Adverse prognostic impact on fetal outcome was noted in the studies conducted by Sucak ${ }^{7}$ and Asicioglu et al. ${ }^{8}$ However this was contradicted by other studies. ${ }^{3,9,10}$ The most common fetal complication was meconium stained amniotic fluid, seen in $34.2 \%$ cases which is comparable to other studies. ${ }^{11}$ This might be due to intra-operative fetal hypoxia caused by strong uterine contraction, deeply impacted fetal head and longer duration of second stage labor.

Neonatal Intensive Care Unit admission rate of $4.5 \%$ and nursery admission rate of 15.3 seen in our study is consistent with published literature. ${ }^{12}$ This was mostly due to newborns requiring septic screening and intravenous antibiotics.

Fresh still birth and perinatal deaths were recorded $23(4.9 \%)$ and $7(1.5 \%)$, respectively in a study. ${ }^{13}$ while we had only one fresh stillbirth and one early neonatal death. Similarly, the same study ${ }^{13}$ reported 37 (6.6\%) cases with Apgar score less than five at five minutes while only 13 (9.0\%) of our babies had an Apgar score of $<7$ at $5 \mathrm{~min}$.

The Royal College of Obstetricians and Gynaecologists in the UK suggests that a consultant be present at all secondstage CS to make an informed decision and to reduce complications arising from such operations. This is not possible in under resourced countries but experienced and trained medical officersmay help to assist in decision making and perform instrumental deliveries and second-stage CS, thereby minimisingthematernal and fetalcomplications.

The limitation of this study is its retrospective nature. Therefore, any suggestions madeshould be taken with caution.

\section{CONCLUSIONS}

Cesarean section in full cervical dilation is an undesirable situation associated with maternal and fetal 
complications. It can be avoided by careful judgement for cephalopelvic disproportion, attendance of skilled health care provider during labor and deliveries and implementation of effective instrumental delivery leading to a better fetomaternaloutcome.The focus should be on ensuring normal progression of labor, proper use of the partogram, pain relief measures, oxytocin augmentation and the promotion of effective pushing techniques.

\section{REFERENCES}

1. Thomas J, Paranjothy S. National sentinel caesarean section audit report. Royal College of Obstetricians and Gynaecologists Clinical Effectiveness Support Unit. London: RCOG Press. 2001 Oct;200(1):43.

2. Vousden N, Cargill Z, Briley A,Tydeman, G, Shennan AH. Caesarean section at full dilatation: incidence, impact and current management. The Obstetrician \&Gynaecologist 2014;16:199-205. FullText

3. Allen VM, O'Connell CM, BaskettTF. Maternal and perinatal morbidity of caesarean delivery at full cervical dilatation compared with caesarean delivery in the first stage of labour. BJOG. 2005;112(7):986-90. FullText

4. Unterscheider J, McMenamin M, Cullinane F. Rising rates of caesarean deliveries at full cervical dilatation: a concerning trend. Eur J Obstet Gynecol Reprod Biol. 2011;157(2):141-4. ScienceDirect

5. Babre VM, Bendre KR, Niyogi G. Review of caesarean sections at full dilatation. Int J Reprod Contracept Obstet Gynecol. 2017;6(6):2491-3. [Link]

6. Shahla Baloch, MeharunnissaKhaskheli, Imdad A. Khushk, AneelaSheeba. Frequency of Second stage Intervention and its outcome in relation with instrumental vaginal delivery versus caesarean section. J Ayub Med Coll Abbottabad. 2008;20(1):87-90.[Link]

7. Sucak A, Celen S, Akbaba E, Soysal S, Moraloglu O, Danisman N. Comparison of nulliparas undergoing cesarean section in first and second stages of labour: a prospective study in a tertiary teaching hospital. Obstet Gynecol Int. 2011.986506. [Link]
8. Asıcıoglu O, Güngördük K, Yildirim G, Asıcıoglu BB, Güngördük ÖÇ, Ark C, Günay T, Yenigül N. Second-stage vs first-stage caesarean delivery: Comparison of maternal and perinatal outcomes. Journal of Obstetrics and Gynaecology. 2014;34(7):598-604. [Link]

9. Selo-Ojeme D, Sathiyathasan S, Fayyaz M. Caesarean delivery at full cervical dilatation versus caesarean delivery in the first stage of labour: comparison of maternal and perinatal morbidity. Archives of gynecology and obstetrics. 2008;278(3):245-9.[Link]

10. Alexander JM, Leveno KJ, Rouse DJ, Landon MB, Gilbert S, Spong CY, Varner MW, Moawad AH, Caritis SN, Harper M, Wapner RJ. Comparison of maternal and infant outcomes from primary cesarean delivery during the second compared with first stage of labor. Obstetrics \& Gynecology. 2007;109(4):917-21.[Link]

11. Jayaram J, Mahendra G, Vijayalakshmi S. Fetomaternal Outcome in Cesarean Sections Done in Second Stage of Labor. Indian Journal of Obstetrics and Gynecology Research. 2016;3(1):51-4.

12. Davis G, Fleming T, Ford K, Mouawad MR, Ludlow J. Caesarean section at full cervical dilatation. Australian and New Zealand Journal of Obstetrics and Gynaecology. 2015;55(6):565-71. [FullText]

13. Umbeli T, Salah Ismail, Kunna A, Elmahgoub A, Nasr A, Rabaa A. Maternal and neonatal complications associated with caesarean section in the second stage of labour at Omdurman maternity hospital during 2012-2013. Merit Research Journal of Medicine and Medical Sciences. 2014;2(10):225-8. [Link] 